$\S=-1$

\title{
Hydraulic System with the Automatic Regulator of Liquid Flow and Investigation of its Operation
}

\author{
Farit D. Bairamov ${ }^{1}$, Bulat F. Bairamov ${ }^{2}$ \\ ${ }^{1}$ Kazan Federal University, Naberezhnochelninsky Institute \\ ${ }^{2}$ Kazan Federal University, Naberezhnochelninsky Institute \\ *Corresponding author E-mail: bbairamov@gmail.com
}

Tel.: 89656132209

\begin{abstract}
Ensuring functioning of a hydraulic system requires the liquid consumption regulation given to the consumer. In hydraulic actuators by change of the liquid consumption regulation of the movement speed of an output link of the hydraulic engine is carried out: the movement speeds of the hydraulic cylinder piston or rotation frequency of a hydraulic motor shaft. In this work with the purpose of increase in efficiency of a hydraulic system and hydraulic actuators it is offered to use in them the most economical way of the liquid consumption regulation due to change of shaft rotation frequency of the pump engine. Schematic diagrams of a hydraulic system and hydraulic actuators with the automatic regulator of the liquid consumption are developed. For the drive of the pump the electric motor of alternating current is used. Shaft rotation frequency of the electric motor is regulated by the frequency converter.

The linearized equations of dynamics of separate links of a hydraulic system from which after an exception of some variables the equations of dynamics of system in general are received are worked out. All equations are written down in a dimensionless form in relative deviations from a nominal operating mode of system. By consideration of hydraulic actuators the equation of dynamics of the hydraulic engine is in addition considered: hydraulic motor or hydraulic cylinder. At the same time the equations of dynamics of the pipeline and the hydraulic engine are replaced with one equation describing dynamics of the integrated link "the pipeline + the hydraulic engine".

The research of stability and accuracy of functioning of a hydraulic system and hydraulic actuators is conducted. Accuracy of functioning is understood as the requirement that deviations of a consumption of the given liquid from its calculated value remained in in advance set limits. Stability conditions are received on the basis of Rauss - Gurvitz criterion, and for the solution of a problem of accuracy of functioning the method of functions of Lyapunov is used. The results received in article can be used at design of various adjustable hydraulic systems and hydraulic actuators for the rational choice of values of the parameters providing stability and accuracy of their functioning.
\end{abstract}

Keywords: hydraulic system, hydraulic actuator, the liquid consumption regulation, dynamics equation, stability and accuracy of functioning.

\section{Introduction}

In mechanical engineering often there is a need of the liquid consumption regulation for hydraulic systems. In hydraulic actuators by change of the liquid consumption speed control of the movement of an output link of the hydraulic engine is exercised: the movement speed of the hydraulic cylinder piston or rotation frequency of a hydraulic motor shaft. Two ways of regulation of a consumption of the consumed liquid are usually used: throttle and volume. At throttle regulation a part of a stream of the liquid arriving from the pump, passing the consumer, it is unproductively taken away on discharge. At a volume way of regulation the consumption of liquid changes due to change of working volume of the pump. Hydraulic system efficiency with volume regulation is higher, than hydraulic systems with throttle regulation. However pumps with an adjustable volume are significantly more expensive than unregulated.

This work is devoted to a problem of increase in efficiency of hydraulic systems and hydraulic actuators due to application in them the most economical way of regulation of the consumption of liquid which is carried out by change of shaft rotation frequency of the pump engine. Schematic diagrams of a hydraulic system and hydraulic actuators with the automatic regulator of the liquid consumption working by the principle of feedback are developed. For a research of functioning of a hydraulic system at first the linearized equations of dynamics of its separate links out of which, excepting some variables, the equations of dynamics of system in general are brought are worked out. At creation of the equations of dynamics of a hydraulic actuator the equation of dynamics of the hydraulic motor or a hydraulic cylinder is in addition considered.

The research of stability and accuracy of functioning of a hydraulic system and hydraulic actuators is conducted. Stability conditions are received by the Rauss-Gurvitz method, and functioning accuracy conditions - method of functions of Lyapunov. Results of a research can be used at design of various adjustable hydraulic systems for liquid supply to the consumer and hydraulic actuators. Also use in hydraulic systems and hydraulic actuators of the combined power food when the ordinary not wind engine and the environmentally friendly wind turbine [1] with the pumps is used to providing the set consumption of liquid is perspective. At a sufficient speed of wind only the windpipe unit works. If the speed of wind is not enough, then not wind engine which works or in the feed mode is connected, or at a small speed of wind completely provides the set liquid consumption. The rotor wind turbine with a vertical axis of rotation $[2,3]$ which does not demand the navigation system on wind most is suitable for the drive of pumps in 
hydraulic systems, it is silent, safe and, unlike vane wind turbines, it can be placed near settlements and buildings.

\section{Methods}

At creation of the equations of dynamics of a hydraulic system and hydraulic actuators with the regulator of the liquid consumption strict and exact methods of mechanics, hydraulics, hydraulic actuators conventional mathematically and the theory of automatic control are used.

Conditions of asymptotic stability of a hydraulic system and hydraulic actuators are received on the basis of Rauss-Gurvitz criterion, and for the solution of a task on the accuracy of their functioning the method of functions of Lyapunov are used.

\section{Results and Discussion}

\subsection{Hydraulic System for Liquid Supply}

In fig. 1 the schematic diagram of a hydraulic system with the automatic regulator of the liquid consumption is submitted. It consists of the electric motor of alternating current 1 , a hydraulic pump 2 , the pipeline 3 , the consumer 4 , a hydro tank 5 and the regulator of the liquid consumption. The last consists of the sensor of an expense 6 , the differential converter with an elastic membrane 8 , the electromechanical reformative device 9 and the frequency converter of tension 10 .

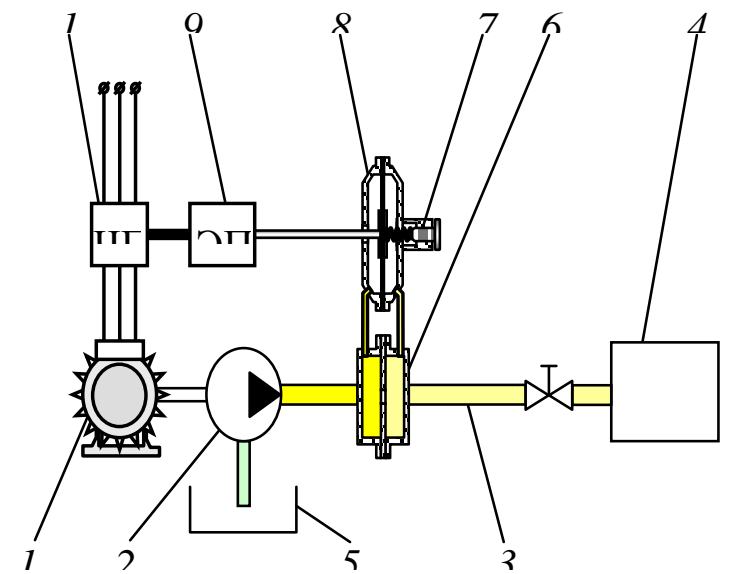

Fig. 1:. The scheme of a hydraulic system with the regulator of the liquid consumption and a power supply from the electric motor

The regulator of an expense works as follows. The expense sensor representing the narrowing diaphragm measures a liquid consumption indirectly through pressure difference. This difference of pressure will be transformed to movement of a membrane of the differential converter. The membrane is connected by a rod with the electromechanical reformative device in which transformation of movement of a rod of a membrane to tension of a direct current proportional to this movement is carried out. This tension operates the frequency converter. Under the influence of the operating tension the frequency converter, changing supply voltage frequency, regulates the speed of rotation of a shaft of the engine. Thus, regulation of a consumption of the given liquid is carried out. The calculated value of an expense is established by the corresponding inhaling of a spring of the control point adjustment 7.

\subsection{Mathematical Model}

Dynamics equations for the electric motor, the pump, the pressure head pipeline, the expense sensor with the differential converter in deviations from a nominal operating mode of a hydraulic system respectively have an appearance [4 - 6]:
$2 \cdot \pi \cdot J_{E} \cdot \frac{d \Delta n_{E}}{d t}=\left(\frac{\partial M_{E}}{\partial n_{E}}\right)_{*} \cdot \Delta n_{E}+\left(\frac{\partial M_{E}}{\partial u}\right)_{*} \cdot \Delta u-\Delta M_{P}$

$\Delta P_{P}=\Delta P_{P 0}-\left(\frac{\partial P_{P}}{\partial G}\right)_{*} \cdot \Delta G+\left(\frac{\partial P_{P}}{\partial n_{E}}\right)_{*} \cdot \Delta n_{E}$

$\frac{l_{P l}}{F_{P l}} \cdot \frac{d \Delta G}{d t}=\Delta P_{P}-\Delta P_{C}-k_{P l} \cdot \Delta G$

$\Delta s=k_{1} \cdot \Delta G$

where $\Delta n_{E}=n_{E}-n_{E_{*}} \quad ; \quad \Delta G=G-G_{*}$ $\Delta P_{P}=P_{P}-P_{P_{*}} \quad . \quad \Delta P_{P 0}=P_{P 0}-P_{P 0_{*}}$

$\Delta s=s-s_{*} ; \Delta P_{C}=P_{C}-P_{C_{*}} ; \Delta u=u-u_{*} ;$ $\Delta M_{P}=M_{P}-M_{P}$ $P_{*}$; the index $*$ hereinafter indicates

values of sizes in the nominal mode; $n_{E}$ - shaft rotation frequency of the electric motor; $G$ - a consumption of the consumed liquid; $P_{P 0} P_{P}$ - pressure on an entrance to the pump and at the exit from it; $P_{C}$ - pressure at the consumer; $u$ - tension of a direct current at the exit of the electromechanical reformative device which is the operating tension for the frequency converter; $S$ shift of a membrane of the differential converter; $k_{1}$ - positive coefficient of proportionality; $J_{E}$ - the total moment of inertia of all bodies which are kinematic tied with an electric motor shaft; $k_{P l}$ - the coefficient characterizing resistance in the pipeline; $l_{P l} F_{P l}$ - length and cross-sectional area of the pipeline;

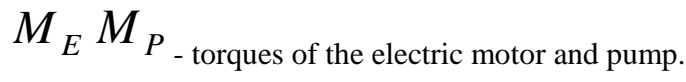

The moment $M_{P}$ is defined by [5] formula $M_{P}=G \cdot\left(P_{P}-P_{P 0}\right) / n_{E} \cdot \eta \cdot \rho$ where $\eta$ - the full efficiency of the pump; $\rho$ - liquid density. Decomposing function $M_{P}$ in a row of Taylor in the neighborhood of the nominal mode and keeping only members of the first degree of rather small increments, we will find:

$M_{P}=\left(\frac{\partial M_{P}}{\partial n_{E}}\right)_{*} \cdot \Delta n_{E}+\left(\frac{\partial M_{P}}{\partial G}\right)_{*} \cdot \Delta G+\left(\frac{\partial M_{P}}{\partial P_{P}}\right)_{*} \cdot\left(\Delta P_{P}-\Delta P_{P 0}\right)$.

The electromechanical reformative device will transform moving of a rod of a membrane to tension of a direct current. Potentiometers (wire, metal-film, semiconductor), inductosin, etc. can be applied to transformation of linear movements to an electric signal, for example, [4]. We will consider the electromechanical reformative device as an intensifying link which dynamics taking into account negative feedback is described by the following equation in deviations: 


$$
\Delta u=-k_{2} \cdot \Delta s
$$

where $k_{2}$ - positive coefficient of proportionality.

The minus sign in (6) means that at the membrane rod shift corresponding to increase in the liquid consumption in the pipeline, the operating tension leads to reduction of shaft rotation frequency of the engine.

In the nominal mode $M_{E_{*}}=M_{P_{*}}=$ const constants, $s=\Delta u=0$ and the regulator of the liquid consumption is in neutral situation.

Further we will assume everywhere $\Delta P_{P 0}=0$, i.e. we neglect indignations of pressure on an entrance to the pump.

The system (1) - (6) describes dynamics of a hydraulic system with the expense regulator. Excepting variables $\Delta P_{P} \Delta u \Delta s$, we will write down this system in relative deviations in a dimensionless form:

а) $T_{E} \cdot \frac{d n}{d t}=-a_{1} \cdot n-a_{2} \cdot g$
б) $T_{P l} \cdot \frac{d g}{d t}=a_{3} \cdot n-a_{4} \cdot g-\frac{P_{C_{*}}}{P_{P_{*}}} \cdot p_{C}$

$n=\frac{\Delta n_{E}}{n_{E_{*}}} g=\frac{\Delta G}{G_{*}} p_{C}=\frac{\Delta P_{C}}{P_{C_{*}}}$;

$a_{1}=\left[-\left(\frac{\partial M_{E}}{\partial n_{E}}\right)_{*}+\left(\frac{\partial M_{P}}{\partial n_{E}}\right)_{*}+\left(\frac{\partial M_{P}}{\partial P_{P}}\right)_{*} \cdot\left(\frac{\partial P_{P}}{\partial n_{E}}\right)_{*}\right] \cdot \frac{n_{E_{*}}}{M_{E_{*}}}$

$a_{2}=\left[\left(\frac{\partial M_{P}}{\partial G}\right)_{*}+\left(\frac{\partial M_{P}}{\partial P_{P}}\right)_{*} \cdot\left(\frac{\partial P_{P}}{\partial G}\right)_{*}\right] \cdot \frac{G_{*}}{M_{E_{*}}}$

$a_{3}=\left(\frac{\partial P_{P}}{\partial n_{E}}\right)_{*} \cdot \frac{n_{E_{*}}}{P_{P_{*}}}$

$a_{4}=\left[\left(\frac{\partial P_{P}}{\partial G}\right)_{*}+k_{P l}\right] \cdot \frac{G_{*}}{P_{P_{*}}}$

$T_{E}=\frac{2 \cdot \pi \cdot J_{E} \cdot n_{E *}}{M_{E_{*}}} \quad T_{P l}=\frac{l_{P l}}{F_{P l}} \cdot \frac{G_{*}}{P_{P_{*}}}$.

Here $T_{E}, T_{P l}$ - constants of time of the electric motor and pipeline, other sizes - dimensionless coefficients.

Stability. Let's write down system (7) without external indignations, i.e. considering that $p_{C}=0$, in a look:

$$
\begin{aligned}
& \frac{d n}{d t}=-\frac{a_{1}}{T_{E}} \cdot n-\frac{a_{2}}{T_{E}} \cdot g ; \\
& \frac{d g}{d t}=\frac{a_{3}}{T_{P l}} \cdot n-\frac{a_{4}}{T_{P l}} \cdot g,
\end{aligned}
$$

Characteristic equation of the system (8):

$\lambda^{2}+\frac{a_{1} \cdot T_{P l}+a_{4} \cdot T_{E}}{T_{E} \cdot T_{P l}} \cdot \lambda+\frac{a_{1} \cdot a_{4}+a_{2} \cdot a_{3}}{T_{E} \cdot T_{P l}}=0$

will have roots with negative material parts when performing inequalities

$a_{1} \cdot T_{P l}+a_{4} \cdot T_{E}>0, \quad a_{1} \cdot a_{4}+a_{2} \cdot a_{3}>0$

which, according to Rauss-Gurvitz criterion, are necessary and sufficient conditions of asymptotic stability of system (8), i.e. a nominal operating mode of a hydraulic system.

Functioning accuracy. Specifications of functioning of a hydraulic system often demand that liquid consumption deviations from its calculated value remained in the set limits. In this regard we will consider a task: to provide inequality performance

$$
|g(t)|<H_{2}, \quad t>0
$$

for any solution of system (8) with initial data from area

$|n(0)|<H_{10}, \quad|g(0)|<H_{20}$,

where $H_{10} H_{20}, H_{2}\left(H_{2}>H_{20}\right)$ - the set positive numbers.

For the solution of this task we use a method of functions of Lyapunov [7 - 9].

When performing conditions of asymptotic stability (9) for system (8) it is possible to construct [7] definitely positive function of Lyapunov in the form of a square form:

$$
V=q_{11} \cdot n^{2}+2 \cdot q_{12} \cdot n \cdot g+q_{22} \cdot g^{2}
$$

meeting a condition

$$
\frac{\partial V}{\partial n} \cdot \frac{\left(a_{1} \cdot n-a_{2} \cdot g\right)}{T_{E}}+\frac{\partial V}{\partial g} \cdot \frac{\left(a_{3} \cdot n-a_{4} \cdot g\right)}{T_{P l}}=-2 \cdot\left(n^{2}+g^{2}\right)
$$

Painting obviously derivative $\partial V / \partial n \partial V / \partial g$ and equating coefficients at similar members, from (13) we will receive the following equations for definition $q_{11} q_{12} q_{22}$ :

$\frac{a_{1}}{T_{E}} \cdot q_{11}+\frac{a_{3}}{T_{P l}} \cdot q_{12}=-1$

$\frac{a_{2}}{T_{E}} \cdot q_{12}+\frac{a_{4}}{T_{P l}} \cdot q_{22}=1$

$-\frac{a_{2}}{T_{E}} \cdot q_{11}+\left(\frac{a_{1}}{T_{E}}-\frac{a_{4}}{T_{P l}}\right) \cdot q_{12}+\frac{a_{3}}{T_{P l}} \cdot q_{22}=0$

From here

$$
\begin{aligned}
& q_{11}=\frac{1}{\Delta_{0} \cdot T_{E} \cdot T_{P l}^{2}} \cdot\left[a_{4} \cdot\left(a_{4} \cdot T_{E}-a_{1} \cdot T_{P l}\right)+a_{3} \cdot\left(a_{2} \cdot T_{P l}+a_{3} \cdot T_{E}\right)\right] \\
& q_{12}=\frac{1}{\Delta_{0} \cdot T_{E} \cdot T_{P l}} \cdot\left(a_{1} \cdot a_{3}-a_{2} \cdot a_{4}\right)
\end{aligned}
$$


$q_{22}=\frac{1}{\Delta_{0} \cdot T_{E}^{2} \cdot T_{P l}} \cdot\left[a_{2} \cdot\left(a_{2} \cdot T_{P l}+a_{3} \cdot T_{E}\right)-a_{1} \cdot\left(a_{4} \cdot T_{E}+a_{1} \cdot T_{P l}\right)\right]$

where $\Delta_{0}$ is defined by a formu-

$\left[\left(a_{2} \cdot a_{3}-a_{1} \cdot a_{4}\right) \cdot\left(a_{4} \cdot T_{E}-a_{1} \cdot T_{P l}\right)\right] /\left(T_{E}^{2} \cdot T_{P l}^{2}\right)$ and is positive owing to (9) size.

The condition (10) will be satisfied if

$q_{11} \cdot H_{10}^{2}+2 \cdot\left|q_{12}\right| \cdot H_{10} \cdot H_{20}+q_{22} \cdot H_{20}^{2} \leq \frac{\Delta \cdot H_{2}^{2}}{\Delta_{2}}$

where $\Delta=\operatorname{det} Q ; Q=\left[q_{i j}\right]_{\text {- matrix of a form }}$

(12); $\Delta=q_{11} \cdot q_{22}-q_{12}^{2}>0 ; \Delta_{2}>0$ - addition

to $q_{22}$ a matrix element $q_{22} Q: \Delta_{2}=q_{11}$.

Really, definitely positive form (12) allows [10] assessment

$\frac{\Delta n^{2}}{\Delta_{1}} \leq V ; \quad \frac{\Delta g^{2}}{\Delta_{2}} \leq V$

where $\Delta_{1}>0$ - addition to $q_{11}$ a matrix ele${ }_{\text {ment }} q_{11} \Delta_{1}=q_{22}$.

Performance of a condition (10) follows from a chain of inequalities

$\frac{\Delta g^{2}}{\Delta_{2}} \leq V(t)<V(0) \leq q_{11} \cdot n^{2}(0)+2 \cdot\left|q_{12}\right| \cdot|n(0)| \cdot|g(0)|+$

$q_{22} \cdot g^{2}(0)<q_{11} \cdot H_{10}^{2}++2 \cdot\left|q_{12}\right| \cdot H_{10} \cdot H_{20}+q_{22} \cdot H_{20}^{2} \leq \frac{\Delta \cdot H_{2}^{2}}{\Delta_{2}}$

which owing to (12) - (15) take place on any solution of the equations (8) coming from the area (11).

Thus, at satisfaction (9), (14) asymptotic stability and accuracy of functioning of a hydraulic system is provided to conditions (8).

Hydraulic actuator with the hydraulic motor. In this hydraulic actuator a consumer of liquid is the hydraulic motor (fig. 2).

Therefore pressure $p_{C}$ in the equation (7b) of the pipeline at the same time is pressure on an entrance to the hydraulic motor.

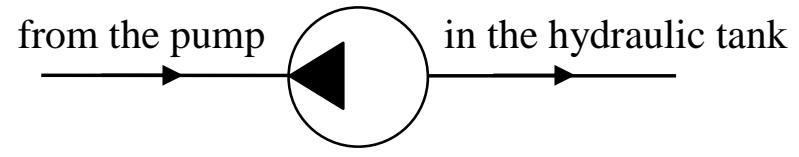

Fig. 2. Hydraulic motor

The equation of dynamics of the hydraulic motor in deviations from a nominal operating mode has an appearance:

$2 \cdot \pi \cdot J_{H m} \cdot \frac{d \Delta n_{H m}}{d t}=\Delta M_{H m}-\Delta M_{R}$

where $\quad \Delta n_{H m}=n_{H m}-n_{H m_{*}}$

$\Delta M_{H m}=M_{H m}-M_{H m_{*}} ; \Delta M_{R}=M_{R}-M_{R_{*}} ;$

$n_{H m}$ - rotation frequency of a hydraulic motor shaft; $J_{H m}$ - the total moment of inertia of the bodies tied with rotation of a hydraulic motor shaft; ${ }^{M} H$ - hydraulic motor torque; ${ }^{M}{ }_{R}$ - the external moment of resistance on a hydraulic motor shaft.

From expression for the torque of the hydraulic motor [5]: $M_{H m}=\frac{W_{H m} \cdot\left(P_{C}-P_{C 0}\right) \cdot \eta_{M}}{2 \cdot \pi}{ }_{\text {where }} W_{H m}, \eta_{M}$ - the working volume and mechanical efficiency of the hydraulic motor; $P_{C 0}$ - pressure at the exit from it, we have

$\Delta M_{H m}=\frac{W_{H m} \cdot \eta_{M}}{2 \cdot \pi} \cdot\left(\Delta P_{C}-\Delta P_{C 0}\right)$

Let's assume further that $\Delta P_{C 0}=0$, i.e. at the exit from the hydraulic engine we consider pressure constant.

The liquid consumption via the hydraulic motor is connected with the rotation frequency of its shaft a formula [5] $G=W_{H m} \cdot \rho \cdot n_{H m} / \eta_{O}$ where $\eta_{O}$ - the volume efficiency of the hydraulic motor. From here we will receive

$$
\Delta n_{H m}=\frac{\eta_{O}}{W_{H m} \cdot \rho} \cdot \Delta G
$$

Let's substitute (17), (18) in (16). We will term by term divide the received equation in which each composed will have dimension of the moment on ${ }^{H m_{*}}$. Besides, we will pass to relative deviations. As a result the equation of dynamics of the hydraulic motor will register in a dimensionless form:

$T_{H m} \cdot \frac{d g}{d t}=\frac{W_{H m} \cdot \eta_{M}}{2 \cdot \pi \cdot M_{H m_{*}}} \cdot P_{C_{*}} \cdot p_{C}-m_{R}$

$m_{R}=\frac{M_{R}}{M_{H m_{*}}} ; T_{H m}$ - hydraulic motor time

con-

$$
T_{H m}=\frac{2 \cdot \pi \cdot J_{H m} \cdot \eta_{O}}{W_{H m} \cdot \rho} \cdot \frac{G_{*}}{M_{H m_{*}}}
$$

Apparently from the equations (7b), (19) condition of the pipeline and the hydraulic motor are defined by the same size - a liquid consumption. Therefore these two links can be united in one. We will receive the equation of dynamics of the pipeline with the hydraulic motor from the equations (7b), (19), excluding from

them pressure $p_{C}$ on an entrance to the hydraulic motor playing a role of internal communication between these two links:

$T_{P l H m} \cdot \frac{d g}{d t}=a_{3} \cdot n-a_{4} \cdot g-\frac{2 \cdot \pi}{W_{H m} \cdot \eta_{M}} \cdot \frac{M_{H m_{*}}}{G_{*}} \cdot m_{R}$

where $T_{P l H m}$ - a pipeline time constant with the hydraulic mo-

$$
T_{P l H m}=T_{P l}+T_{H m} \cdot \frac{2 \cdot \pi \cdot M_{H m_{*}}}{W_{H m} \cdot \eta_{M} \cdot P_{P_{*}}}
$$

Thus, dynamics of a hydraulic actuator with the hydraulic motor is described by the equations (7a), (20). Stability conditions and the 
accuracy of functioning of a hydraulic actuator at $m_{R}=0$ remain in the form of inequalities (9) and (14) in which $T_{P l}$ it is replaced on $T_{P l H m}$.

\subsection{Hydraulic Actuator with A Hydraulic Cylinder}

Here as the consumer of liquid the hydraulic cylinder 1 performs with the hydraulic valve 2 (fig. 3 ).

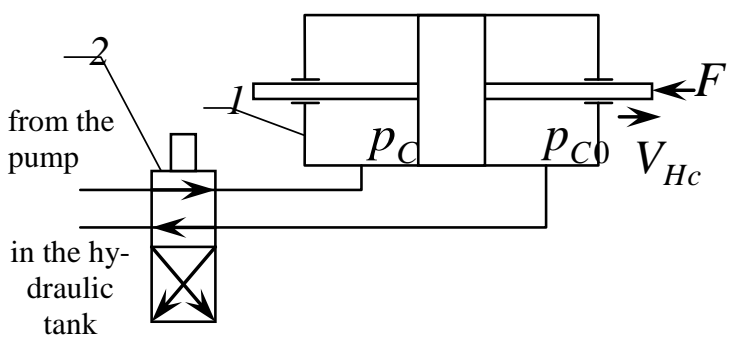

Fig. 3:. A hydraulic cylinder with the hydraulic valve

Let the hydraulic cylinder piston move with a speed to the right $V_{H c}$, overcoming external loading on a rod $F$. Neglecting losses on friction, we will write down the equation of dynamics of the hydraulic cylinder piston in deviations from a nominal operating mode:

$m \cdot \frac{d \Delta V_{H c}}{d t}=\left(\Delta P_{C}-\Delta P_{C 0}\right) \cdot \sigma-\Delta F$

where $\Delta V_{H c}=V_{H c}-V_{H c_{*}} ; \Delta P_{C}=P_{C}-P_{C_{*}}$; $\Delta P_{C 0}=P_{C 0}-P_{C 0_{*}} ; \Delta F=F-F_{*} ; P_{C} P_{C 0}$ pressure in the left and right cavities of a hydraulic cylinder;

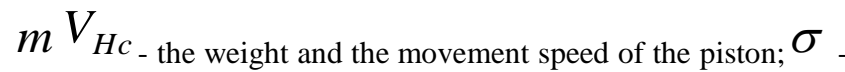
ring area of the piston.

Let's assume further that $\Delta P_{C 0}=0$. Everyone composed the equations (21), dimensional forces, we will divide on $F_{*}$ and we will pass to relative deviations. As a result we will receive the dimensionless equation of dynamics of a hydraulic cylinder in a look:

$$
\frac{m \cdot V_{H c_{*}}}{F_{*}} \cdot \frac{d v_{H c}}{d t}=\frac{\sigma}{F_{*}} \cdot P_{C_{*}} \cdot p_{C}-f
$$

$v_{\text {where }}=\frac{\Delta V_{H c}}{V_{H c_{*}}} p_{C}=\frac{\Delta P_{C}}{P_{C_{*}}} f=\frac{\Delta F}{F_{*}}$.

The liquid consumption through a hydraulic cylinder is connected with piston speed a formula $G=\sigma \cdot \rho \cdot V_{H c}$. From here, we will receive

$$
\Delta V_{H c}=\frac{G_{*}}{\sigma \cdot \rho \cdot V_{H c_{*}}} \cdot \Delta G
$$

Taking into account (23) equation (22) takes a form:
$T_{H c} \cdot \frac{d g}{d t}=\frac{\sigma}{F_{*}} \cdot P_{C_{*}} \cdot p_{C}-f$

where $T_{H c}$ - a hydraulic cylinder time con-

$$
T_{H c}=\frac{m}{\sigma \cdot \rho} \cdot \frac{G_{*}}{F_{*}} .
$$

As well as in case of a hydraulic actuator with the hydraulic motor, the pipeline equations (7b) and a hydraulic cylinder (24), excluding from them pressure $p_{C}$, we will replace with one equation:

$$
T_{P l H c} \cdot \frac{d g}{d t}=a_{3} \cdot n-a_{4} \cdot g-\frac{F_{*}}{\sigma \cdot P_{P_{*}}} \cdot f
$$

where $T_{P l H c}$ - a pipeline time constant with a hydraulic cylin-

$$
T_{P l H c}=T_{P l}+T_{H c} \cdot \frac{F_{*}}{\sigma \cdot P_{P_{*}}} \text {. }
$$

Thus, dynamics of a hydraulic actuator with a hydraulic cylinder is described by the equations (7a), (25). Stability conditions and the accuracy of functioning of a hydraulic actuator at $f=0$ remain in the form of inequalities (9) and (14) in which $T_{P l}$ it is replaced on $T_{P l H c}$.

\section{Conclusions}

1) Stability conditions of a hydraulic system and hydraulic actuators in the form of inequalities connecting coefficients (parameters) of system, and a condition of accuracy of their functioning in the form of inequalities connecting system parameters with the numbers limiting deviations of variables of a state from their calculated values are received.

2) It is shown what by consideration of a hydraulic actuator with the hydraulic motor of the equation of dynamics of the pipeline (7b) and the hydraulic motor (19) can be reduced to one equation (20) describing dynamics of the integrated link "the pipeline + the hydraulic motor". Similarly, in case of a hydraulic actuator with a hydraulic cylinder of the equation of dynamics of the pipeline (7b) and a hydraulic cylinder (24) come down to one equation (25).

3) Dynamics equations, stability conditions and functioning accuracy for a hydraulic system and hydraulic actuators have an identical appearance (7), (9), (14) and differ only with values of constants of time of the pipeline and the integrated link "the pipeline + the hydraulic engine". In case of the pipeline with the hydraulic motor or a hydraulic cylinder $T_{P l}$ it is replaced $T_{\text {on }} T_{P l H c}$ respectively.

\section{Summary}

1. Schematic diagrams of a hydraulic system and hydraulic actuators with automatic control of the liquid consumption due to change of shaft rotation frequency of the electric motor of the pump are developed. Such regulation, being the most economic, increases efficiency of a hydraulic system and hydraulic actuators. 
2. Conditions (9), (14) can be used at design of various adjustable hydraulic systems for liquid supply to the consumer and hydraulic actuators for the rational choice of values of the parameters providing stability and the set accuracy of their functioning.

\section{Acknowledgement}

The work is performed according to the Russian Government Program of Competitive Growth of Kazan Federal University.

\section{References}

[1] T.S. Jagadevlah, R.T. Smith, "Generation schemes for wind power plants", paper presented in the 10th Intersociety Energy Conversion Engineering Conference (IECEC), Newark.: Delaware, August, 1975.

[2] F.D. Bairamov, I.G. Mardamshin, "Investigation of operation of hydraulic systems with wind pumping units", Russian Aeronautics, vol. 51, No. 3, pp. 314-320, 2008.

[3] Farit D. Bairamov, Bulat F. Bairamov, "About hybrid system stability with distributed and concentrated parameters", Helix, vol. 8, No. 1, pp. 2493-2498, 2018.

[4] N.N. Ivashchenko, "Automatic control", M.: Mechanical engineering, 736 pages, 1978.

[5] A.V. Lepeshkin, A.A. Mikhaylin, A.A. Sheypak, "Hydraulics and hydropneumatic actuator. In 2 parts. Part 2. Hydraulic machines and hydropneumatic actuator", M.: MGIU, 352 pages, 2005.

[6] A.M. Abdullina, F.D. Bayramov, B.F. Bayramov, "A hydraulic actuator with the operated electric motor of the pump and a research of its dynamics", the Scientific and technical bulletin of the Volga region, No. 2, S. 65-68, 2014.

[7] I.G. Malkin, "Theory of stability of the movement", M.: Science, 530 pages, 1966.

[8] K. Peiffer, N. Rouche, "Liapunov's second method applied to partial stability", J. de Mech., vol. 8, No. 2, pp. 323-334, 1969.

[9] T. Yoshizawa, "Stability theory by Lyapunov's second method", Tokyo: Math. Soc. Jap., 223 p., 1966.

[10] F.D. Bayramov, B.F. Bayramov, N.S. Galimov, A.R. Fardeev, "Technological processes with the combined power food: mechanics, management, automation", Kazan: Publishing house of the Kazan university, 342 pages, 2016. 